\title{
The Approach of Genetic Algorithms Application on Reactive Power Optimization of Electric Power Systems
}

\author{
RuiJin ZHU ${ }^{1, a}$ \\ ${ }^{1}$ XiZang agriculture and Animal Husbandry College, Linzhi City 860000,China \\ a zrj6506@126.com
}

\begin{abstract}
Keywords: Electric Power Systems; Genetic Algorithms; Reactive Power Optimization
\end{abstract}
\begin{abstract}
With the rapid growth of China's economy and the development of industry, the demand of power quality in various departments of national economy is more and more strict. In the power system, reactive power plays a special role. The research on reactive power optimization of power system has significant practical significance for reducing the extra active power consumption and improving the voltage operation level caused by the unreasonable allocation of reactive power. Using the improved genetic algorithm proposed in this paper, the standard test system is used to simulate the reactive power optimization, and the optimization results of the simple genetic algorithm and the improved genetic algorithm are compared. The simulation results show that the proposed algorithm is feasible and effective, and the improved genetic algorithm has lower active network loss and better global convergence performance and convergence speed.
\end{abstract}

\section{Introduction}

With the rapid development of China's national economy and power industry, the total installed capacity of power generation in China and the demand for electricity in all walks of life are increasing at an unprecedented rate. At present, China grid scale has surpassed the United States ranked first in the world, the installed power generating capacity continues to rank second in the world, the power system security, reliability, economy and resource allocation capabilities improve, basically meet the electricity needs of economic and social development [1-2]. With the continuous growth of power generation capacity, power generation and social electricity consumption, higher requirements are put forward for the safety of power grid and power quality. Therefore, the security, economic and stable operation of power system is becoming more and more important. Reactive power optimization is an effective means to ensure the safe, economic and stable operation of power system, and is an important measure to improve the quality of system voltage [3].

In order to improve the quality of voltage and improve power system and reduce the power loss of the whole system, ensure the economic benefit of power system, it needs the appropriate transmission power, reactive power, power distribution network reactive power compensation device and adjustable pressure control device, to ensure adequate system reactive power reserve capacity, voltage level the stability of the entire system, protection of electrical equipment, to maintain its normal state of operation and improve the economic benefits of equipment operation. At the same time, in the process of transmission and distribution, it is necessary to avoid the long-distance transmission of reactive power and redundant reactive power transmission in the power grid, so as to reduce the active power loss of the system. This is of great significance to realize the security, stability and economic operation of the whole power system. The optimization of reactive power in power system is an optimization problem. It has many kinds of variables, more than one goal, complex mathematical model, large processing scale, and high requirement for real-time algorithm. Especially in recent years, the scale of power system is becoming larger and larger, and the technology is becoming more and more complex. Due to the inherent limitations of the traditional algorithm, it has been unable to adapt to the modern large-scale power system. In recent years, artificial intelligence algorithm has been applied in the field of reactive power 
optimization, among which genetic algorithm is more widely used than other intelligent algorithms. In this paper, genetic algorithm is improved on the basis of the existing research, in order to further improve the speed and accuracy of its solution.

\section{Related work}

Reactive power optimization of power system is a large-scale, dynamic, multi objective, multi constrained nonlinear optimization problem. The optimization process is very complex, and the operation variables include not only continuous variables, but also discrete variables [4]. The reactive power optimization of power system up to now, many domestic and foreign experts and scholars carried out a lot of research work, a series of effective algorithms and mathematical model is proposed to solve the reactive power optimization problem, made a lot of achievements. Especially in recent decades, with the development of modern artificial intelligence algorithms and the rapid development of computer science and technology, people will use these advanced theory to solve the problem of reactive power optimization, provides a new theoretical method for the research and analysis of reactive power optimization [5-6]. So far, the solution methods of reactive power optimization can be divided into two categories: conventional optimization algorithm and modern artificial intelligence algorithm.

\section{Genetic algorithm research for power system operation}

The genetic algorithm is a random search method simulating the evolutionary mechanism proposed in 1970s by American professor J.Holland of the University of Michigan. The algorithm can globally optimal solution space in multi path search problem, and to solve the optimization problem in the mathematical model of operating variables encoding processing, the object of genetic manipulation by the operating variables are converted to digital string, which makes the selection, crossover and mutation genetic operation becomes easy. GA searching direction is directly determined by the objective function, does not need the derivation for the inverse of complex mathematical operations, improves the efficiency of the algorithm, at the same time, GA is adopted to group $\mathrm{N}$ the number of individuals on the unit to carry out optimization, can also handle a large number of patterns, with strong processing line ability, which can fast global convergence. At the same time, because the GA is able to handle the constraints of the optimization problem, the introduction and not too restrictive, more conducive to the algorithm converges to global optimal solution space solution of non-linear programming problem in the solution of reactive power optimization of power system with multiple variables and constraints in this category are wide application. In view of the above characteristics of genetic algorithm, many scholars at home and abroad have done a lot of work in recent years. GA is applied to solve the problem of reactive power optimization in power system, and has achieved some experience and results [7].

The solution steps of the IGA are shown in Figure 1. (1) Initialize the requirements and set number of "catastrophe". (2) Come into being out the initial race. (3) Operate the genetic evolution. (4) Record the maximum and the minimum adaptation value individual in the population. (5) Carry out the catastrophe and preserve the current optimal solution while the difference between the maximum and the minimum adaptation value individual is smaller than a threshold, and return to step (3). Otherwise, take the next step. 


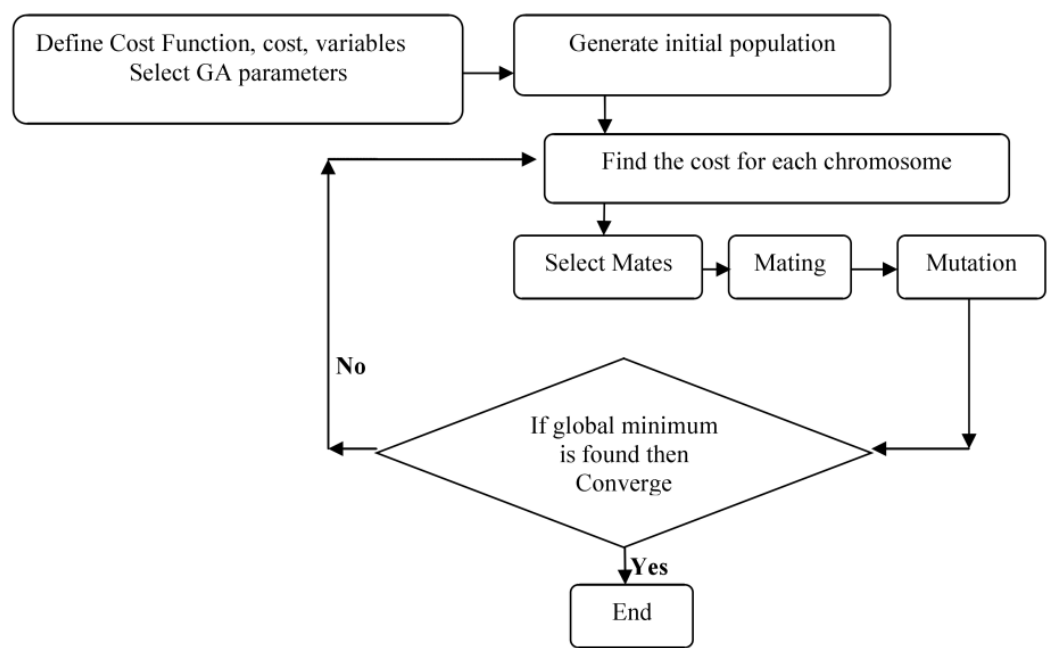

Figure 1. Flow chart for Genetic Algorithm Optimization

Application process of Genetic algorithm for power system operation is shown in Figure 2.

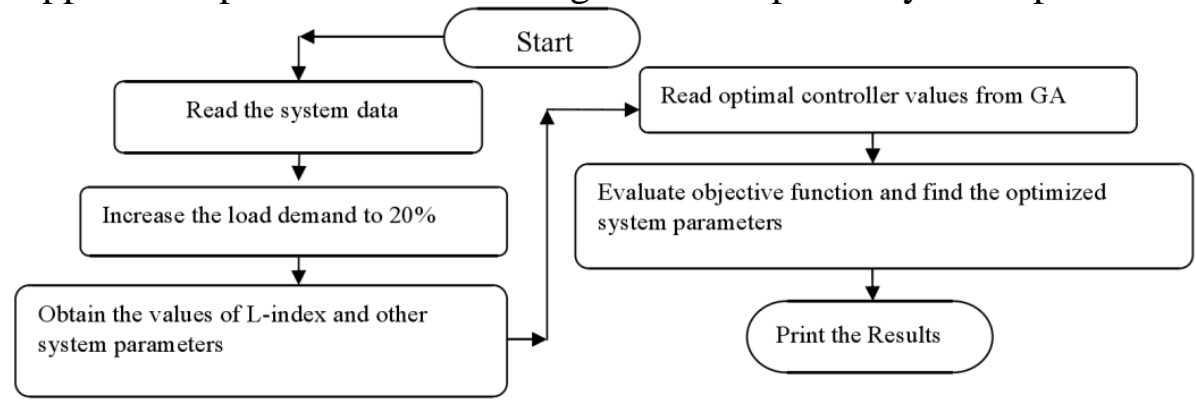

Figure 2. Flow chart for power system operation

\section{Simulation and discussion}

The proposed GA is implemented using the MATLAB platform for RPO. And the calculations are deployed on IEEE 14, 30, 57-bus system. All of the bus voltage ranges of these three benchmark systems are $0.95 \sim 1.1$ (per-unit value). The increment step size of the transformer is given as $1.25 \%$, the lower and upper ratio limit are 0.9 and 1.1 , respectively. And, the upper limit for the account of stunt capacitor is 5, its step size is 0.1. Parameters used in the GA are set as the following: popsize $=50$, maximum generation loop $\mathrm{t}=100$, P C0 $=0.8$, increment step size P cstep 0.002 , $\mathrm{P}$ Cmin $=0.6$, mutation rate $\mathrm{P} \mathrm{m} 0=0.1$, the decrement step size $\mathrm{P}$ mstep $=0.001, \mathrm{P}$ max $=0.2$, penalty coefficient of the voltage $\mathrm{V}=1.05$, penalty coefficient of generator's reactive power $\mathrm{Q}=1.025$. Both of the simple genetic algorithm (SGA) and the improved genetic algorithm (IGA) will be tested on all the three systems for ten times. Data from Table 1 manifest that the optimal value of IEEE 14 system by the improved genetic algorithm (IGA) is 0.126935 , and the reduction ratio is $8.35 \%$, better than the SGA result, 0.129240 . The reduction ratio of IEEE 30 and IEEE57 are $11.44 \%$ and $17.43 \%$, respectively, proving that the optimization performance effect is notable. Figure 3 shows the convergence history of the objective function along the evolutionary generation of the optimal optimization result out of the ten runs, regarding the IEEE 14 bus system. 


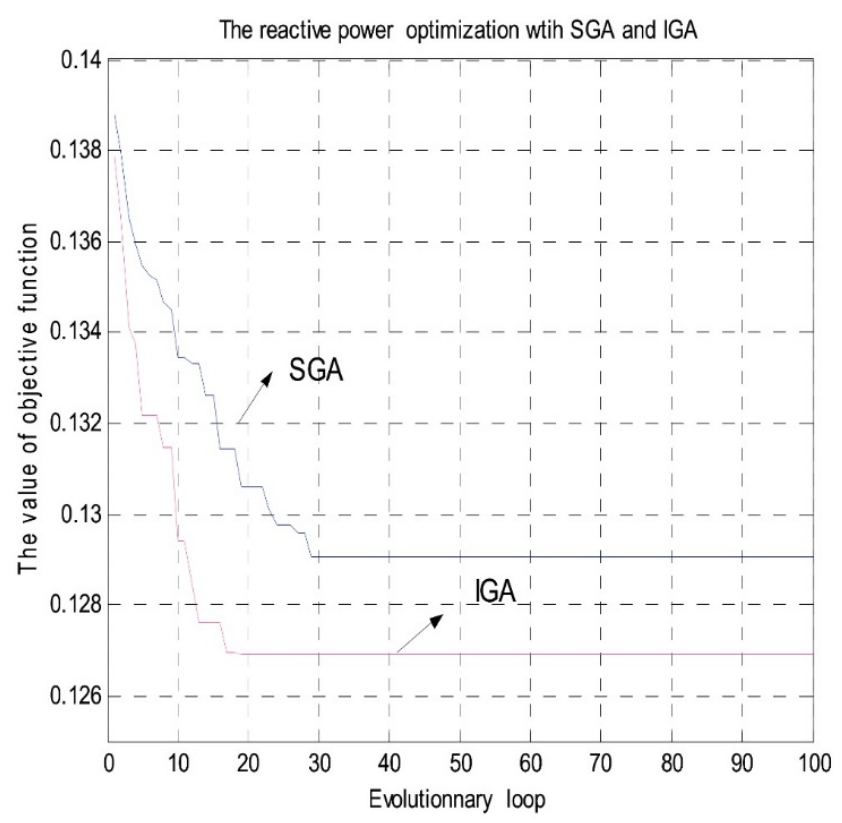

Figure 3. Comparison of the convergence performances of IEEE 14 system

The y-axis refers to the minimum evolutionary value of each generation. As revealed in Figure 3 and 4, the improved GA can more efficiently return smaller optima than the SGA. The comparison indicates that the proposed IGA is feasible and effective in solving the RPO problems.

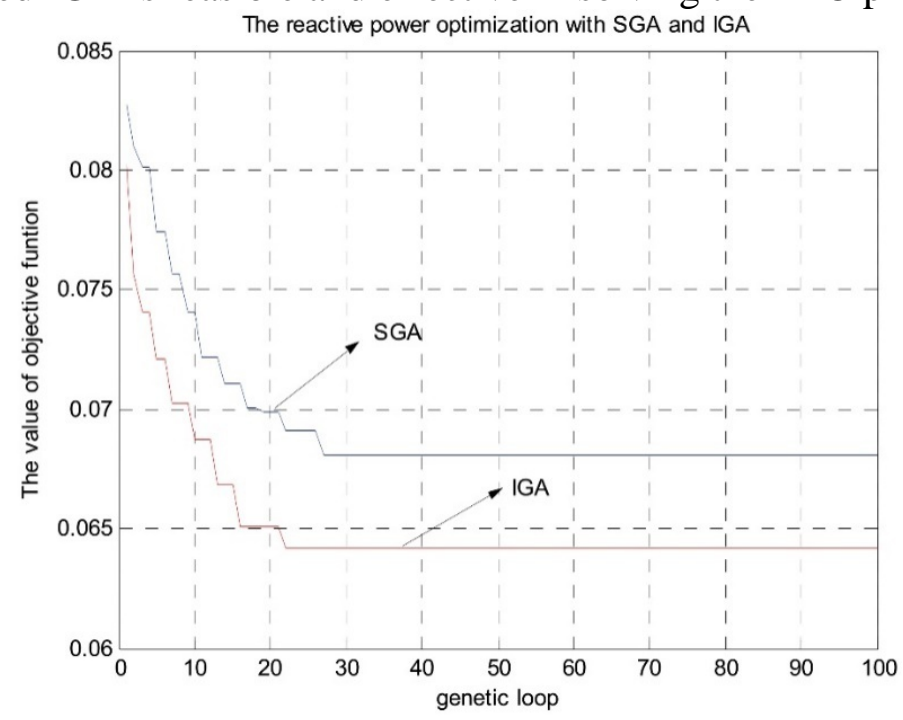

Figure 4. Comparison of the convergence performance of IEEE 30 system

\section{Conclusion}

Reactive power optimization of power system is not only an effective means to ensure the security, stability and economic operation of power system, but also an important measure to effectively reduce the power system network loss and improve the voltage quality. Therefore, the research on reactive power optimization of power system has both theoretical significance and practical application value. Reactive power optimization of power system is a multi-variable, multi constrained and nonlinear mixed programming problem. Its operation variables include continuous variables and discrete variables, and the optimization process is also very complicated. Genetic algorithm is widely used in solving the problem of reactive power optimization because it can search the global optimal solution by multi path, and can solve the discrete and nonlinear problems naturally. Therefore, based on the establishment of the mathematical model of reactive power optimization, an improved genetic algorithm is proposed and applied to solve the reactive power optimization problem, and the effect of reactive power optimization is improved. Using the 
improved genetic algorithm proposed in this paper, the standard test system is used to simulate the reactive power optimization, and the optimization results of the simple genetic algorithm and the improved genetic algorithm are compared. The simulation results show that the proposed algorithm is feasible and effective, and the improved genetic algorithm has lower active network loss and better global convergence performance and convergence speed.

\section{Acknowledgements}

1.XiZang agriculture and Animal Husbandry College High-level Talents Introduction Project (2014DQ-ZN-01).

2.Key research projects of Tibet Autonomous Region for Innovation and Entrepreneur(Grant No.Z2016D01G01/01).

\section{Reference}

[1] Zhu M, Xu Z, Li X, et al. Dynamic Reactive Power Optimization Based on Genetic Algorithms in Power Systems[C]// International Conference on Mechatronics, Control and Electronic Engineering. 2014.

[2] Wang L Y. Research on Reactive Power Optimization Based on Genetic Algorithms in Distribution Network[J]. Applied Mechanics \& Materials, 2014, 644-650(3):2476-2478.

[3] Liu Z, Wang X, Li C. Reactive power optimization in power system based on chaos ant colony algorithm[C]// International Conference on Sustainable Power Generation and Supply, 2009. Supergen. IEEE, 2010:1-4.

[4] Liu B Y, Yang R G, Hui L I, et al. Reactive Power Optimization Based on Chaos Genetic Algorithm in Electric Power Systems[J]. Proceedings of the CSU-EPSA, 2006, 18(5):49-52.

[5] Ma X, Liu R L. Reactive power optimization in power system based on improved niche genetic algorithm[C]// International Conference on Computer Design and Applications. IEEE, 2010:V3-413-V3-416.

[6] Dai C, Chen W, Zhu Y, et al. Reactive power dispatch considering voltage stability with seeker optimization algorithm[J]. Electric Power Systems Research, 2009, 79(10):1462-1471.

[7] Kim H M, Kim J Y, Yoon C D, et al. Optimal Voltage and Reactive Power Control of Local Area Using Genetic Algorithm[C]// International Conference on Knowledge-Based and Intelligent Information and Engineering Systems. Springer, Berlin, Heidelberg, 2006:900-906. 